\title{
Análisis de los factores que determinan la adherencia terapéutica del paciente hipertenso
}

\author{
Inmaculada Zurera Delgado, M $^{\mathrm{a}}$ Teresa Caballero Villarraso, Margarita Ruíz García \\ Unidad de Hipertensión. UGC Nefrología. Hospital Universitario Reina Sofía de Córdoba
}

\section{Resumen}

Introducción: La baja adherencia al tratamiento en enfermedades crónicas como la hipertensión arterial, sigue siendo actualmente un problema latente en nuestro país. A pesar de que la educación sanitaria se viene utilizando en los distintos niveles asistenciales para mejorar la adherencia al tratamiento farmacológico y la adquisición de hábitos de vida saludables, no existen muchas evidencias de su eficacia, especialmente en pacientes hipertensos, refractarios a modificar sus hábitos higiénico-dietéticos.

Objetivo: El propósito de este estudio fue conocer las motivaciones personales, que intervienen en la modificación de los hábitos, adherencia y conocimiento de la enfermedad, en un grupo de pacientes hipertensos.

Metodología: Estudio fenomenológico realizado en el ámbito hospitalario, mediante entrevistas semiestructuradas. La muestra fue de dieciocho pacientes con hipertensión arterial no controlada, asociada a síndrome metabólico y/u obesidad (con IMC >30), durante un periodo de 4 meses en la consulta de UGC Nefrología/ HTA, del HURS de Córdoba.

Resultados: Se hallaron tres categorías temáticas con las que dar respuesta a nuestros objetivos de estudio: Sensibilización acerca del riesgo cardiovascular al que están expuestos y conocimiento de la propia enfermedad, razonando los motivos que impulsan o retraen al paciente hipertenso a adherirse al tratamiento y relación entre cumplimiento del tratamiento farmacológico y no farmacológico.

Conclusiones: La falta de adherencia al tratamiento, se manifiesta por una falta de voluntad entre los pacientes hipertensos refractarios, más que por un problema de información, siendo necesaria una intervención por parte del personal de enfermería enfocado a profundizar en la misma.

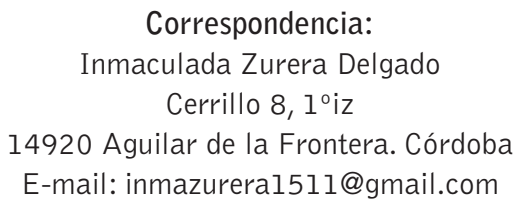

PALABRAS CLAVE

- HIPERTENSIÓN ARTERIAL

- EDUCACIÓN SANITARIA

- ADHERENCIA TERAPÉUTICA

- MEDIDAS HIGIÉNICO-DIETÉTICAS

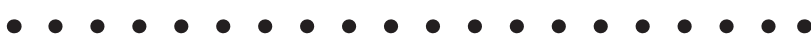
Analysis of the determining factors of therapeutic adherence in hypertensive patients

\begin{abstract}
Introduction: Poor adherence to treatment in chronic diseases such as hypertension currently remains a latent problem in our country. Although the Health Education is been used in different levels of health care to improve adherence to drug therapy and acquisition of healthy lifestyles, there is little evidence of their effectiveness, especially in hypertensive patients, refractory to change their hygienic-dietary habits.

Objective: This study was realized with the aim of learning personal motivations involved in changing habits, adherence and knowledge of the disease in a group of hypertensive patients.

Method: Phenomenological study in the hospital setting, using semi- structured interviews. The sample was made of eighteen patients with uncontrolled levels of blood pressure associated with metabolic syndrome and / or obesity (BMI>30). These patients are been visited by the health care team of Nephrology UGC/Hypertension, in the Hospital Universitario Reina Sofía of Córdoba, since last January.
\end{abstract}

Results: Three thematic categories with which to answer our study objectives, have been found: Raising awareness about cardiovascular risk to which they are exposed and knowledge of the disease itself, justifying the reasons which motivate or retract the hypertensive patient to adhere to treatment, and relationship between compliance pharmacological and non-pharmacological treatment. 
Conclusion: The lack of adherence to treatment is showed up by unwillingness among hypertensive patients refractory, rather than an information problem. For this reason, a nursing intervention focused on solving the problem, is necessary.

\section{KEYWORDS}

- HYPERTENSION

- HEALTH EDUCATION

- ADHERENCE THERAPEUTIC

- HYGIENIC-DIETARY MEASURES

\section{Introducción}

La hipertensión arterial (HTA) es un problema de salud pública de primera magnitud, por ser uno de los factores de riesgo cardiovascular más importante. Su prevalencia se encuentra en constante ascenso y puede derivar en graves complicaciones, especialmente en los sujetos con mal control de las cifras de presión arterial ${ }^{1,2}$.

Se ha estimado que la HTA está relacionada con el $46,4 \%$ de las muertes por enfermedades cerebrovasculares, con el $42 \%$ de las muertes coronarias y con el $25,5 \%$ de las muertes totales, partiendo de la distribución de presión arterial (PA) en la población española de 35 a 64 años, y de los riesgos relativos de muerte establecidos en estudios internacionales ${ }^{3}$.

Sin embargo, solo un $20 \%$ de los pacientes hipertensos con patologías asociadas como las cardiovasculares, cerebrales, renales y diabéticas, tienen la PA controlada dentro de los límites recomendados por la Organización Mundial de la Salud (OMS), siendo este mal control, el principal determinante de la incidencia de enfermedad cardiovascular en España ${ }^{1,2,4}$. Entre las últimas publicaciones de la Sociedad Española de Cardiología, tal y como se muestra en el estudio "Incidence and Prognosis of Resistant Hypertension in Hypertensive Patients" (Daugherty, 2012), de los pacientes con hipertensión incidente donde se inició el tratamiento, uno de cada 50 pacientes desarrollaron hipertensión refractaria. En lo que respecta al control alcanzado, uno de cada 6 pacientes con 3 ó más antihipertensivos, seguían cumpliendo los criterios de HTA refractaria un año después; afirmando que los pacientes con hipertensión refractaria mostraron un mayor riesgo de eventos cardiovasculares 5 .

Por tanto, es importante que la población hipertensa tenga conciencia de la importancia de este factor de riesgo, así como de los beneficios del tratamiento y del control de las cifras que, en definitiva, incidirán en una mayor cantidad y mejor calidad de vida. Es importante además, que la persona hipertensa tenga el máximo de conocimientos sobre su enfermedad y los factores de riesgo asociados, ya que la mayoría de ellos los desconocen, sobretodo en sus inicios. Por ello, es fundamental la implantación de programas de educación sanitaria (ES) en todos los niveles asistenciales, en estos pacientes ${ }^{1}$.

Existen evidencias científicas de que la ES y la intervención de enfermería, como responsable de la misma, son efectivas en el control de las cifras tensionales y en la adherencia terapéutica. Roter et al, tras un metaanálisis de 153 estudios que valoraban la efectividad de diferentes formas de intervención para mejorar el cumplimiento, observó que la HTA precisamente, era una de las enfermedades que se beneficiaba especialmente de cualquiera de ellas $s^{6,7}$.

Sin embargo, la bibliografía médica más reciente, cuyo objetivo de estudio es el cumplimiento terapéutico del paciente hipertenso, ofrece escasas publicaciones; $y$ en ellas se muestra que los niveles de presión arterial y la adherencia al tratamiento siguen siendo bajas, entrando en juego en algunos casos, la inercia clínica profesional ${ }^{8,9}$.

Asimismo, no existe mucha bibliografía que documente la eficacia de las diferentes estrategias de intervención para mejorar el cumplimiento terapéutico, ni en Espa-

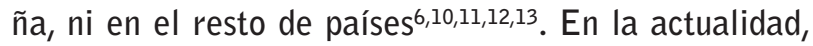
es una realidad que la ES se realiza por la mayoría de los profesionales de enfermería, y en casi todos los niveles asistenciales. Se trata de una competencia poco "explorada" por el personal de enfermería, teniendo en cuenta que la ES, es la base para la buena adherencia al tratamiento en enfermedades crónicas sobre todo; realizándola fundamentalmente, los equipos de Atención Primaria.

No obstante, los resultados indican por el contrario, que una cifra considerable de pacientes hipertensos, a día de hoy, siguen sin controlar sus niveles de presión arterial, y han de ser derivados a una consulta de Atención Especializada para su posterior control. En esta consulta, se someten a estudios más profundos, buscando otras patologías asociadas que contribuyen a aumentar el riesgo cardiovascular, daño renal, etc. Así, es frecuente encontrar pacientes con obesidad o síndrome metabólico, asociados a la HTA, con un IMC $>30$. Además, muchos de ellos son refractarios a las medidas higiénico-dietéticas, y son incapaces de perder peso. 
Precisamente por esto, en la Consulta de la Unidad de HTA de la UGC Nefrología, desde hace unos meses se está llevando a cabo un programa de ES enfocado a modificar los hábitos higiénico-dietéticos, con el fin de controlar la HTA, y que el paciente compren$\mathrm{da}$, que este tipo de tratamiento es tan necesario e importante como el tratamiento farmacológico, que ambos se complementan e incluso, en algunos casos, gracias al tratamiento no farmacológico, la dosis de hipotensores podría reducirse e incluso llegar a no ser necesaria.

Esta intervención, se ha centrado en aquellos aspectos donde había que reforzar los conocimientos de los pacientes y sobre todo, en el refuerzo de cambios de conducta, siendo una intervención individualizada y no estructurada; adaptada en todo momento a las necesidades educativas en esta materia de los pacientes, todos con un problema de sobrepeso importante y con dificultades para la pérdida de peso.

Por todo ello, el presente estudio se ha centrado en indagar en las motivaciones personales de los pacientes, a la hora de modificar o no sus hábitos, adherencia y conocimiento de la enfermedad.

\section{Objetivos}

- El objetivo principal de la investigación fue:

- Conocer las motivaciones personales, que intervienen en la modificación de los hábitos, adherencia y conocimiento de la enfermedad, en un grupo de pacientes hipertensos.

- Los objetivos específicos fueron:

- Evaluar la sensibilización que tienen los pacientes con HTA no controlada, participantes de este estudio, acerca del riesgo cardiovascular al que están expuestos.

- Analizar los puntos débiles a nivel emocional para reforzar en posteriores consultas de enfermería.

- Determinar la relación entre el cumplimiento de las medidas farmacológicas y no farmacológicas.

\section{Metodología}

\section{Diseño}

La metodología elegida para llevar a cabo este estudio ha sido un enfoque cualitativo, ya que gracias a la investigación cualitativa, es posible comprender e interpretar la realidad, los sentimientos, los significados y las acciones de las personas desde su propio punto de vista, siendo por tanto la que mejor se adaptaba a nuestro objetivo de estudio. Se trata de un diseño fenomenológico enfocado en las experiencias individuales y subjetivas de los participantes. Con el propósito de explorar las razones que los impulsan a cambiar sus hábitos de vida y con ello adherirse al tratamiento, tras la intervención educativa, o por el contrario, los motivos que los conducen a no cambiar ${ }^{14}$.

\section{Sujetos de estudio}

\section{Población diana:}

El estudio fue realizado dentro del ámbito hospitalario, contando con la participación de pacientes con HTA no controlada que desde el mes de enero de 2014 han sido derivados a la consulta de UGC Nefrología/HTA (perteneciente a las Consultas Externas) del Hospital Universitario Reina Sofía, procedentes de sus centros de Atención Primaria, para reajustar su tratamiento y con ello controlar los niveles de PA.

\section{Población de estudio:}

Se escogieron aquellos pacientes que cumpliendo los criterios de inclusión, pudiesen aportar más información relevante para lograr responder a los objetivos de nuestro estudio.

Los criterios de inclusión para la participación en el estudio fueron los siguientes:

- Ser hipertenso con niveles de presión arterial mal controlados, ligado a Síndrome Metabólico u obesidad (IMC $>30$ ).

- Haber sido derivado desde Atención Primaria a Especializada a partir de enero de 2014.

- Haber recibido ES por parte de la enfermera de dicha consulta en al menos una ocasión.

\section{Muestra:}

Para la selección de la muestra y el posterior acceso a la misma, la investigadora contó con el apoyo de su tutor en dicho estudio, quien medió con los profesionales que trabajan en dicha consulta, para que el desarrollo del estudio fuese posible. De modo que, una vez que fue acogida la investigadora por los profesionales, y tras asistir en varias ocasiones a la consulta de enfermería en la que se realiza la ES a los pacientes a nivel individual, la investigadora junto con la profesional de enfermería encargada de llevar a cabo el Programa de ES, procedieron a la selección de la muestra, a través de un muestreo no probabilístico, intencional y de conveniencia. 
La muestra elegida fue de veintiséis pacientes, de los cuales solo pudieron ser citados dieciocho, por motivos de diversa índole (imposible contacto telefónico, imposible desplazamiento al lugar propuesto, ingreso hospitalario, problemas familiares...). Hubo mayor número de participantes masculinos, siendo doce hombres y tan solo seis mujeres, en un rango de edad comprendido entre los 50 y 76 años.

\section{Técnicas de recogida de información}

De entre las técnicas disponibles para la recogida de información fue escogida la entrevista, en concreto la entrevista semi-estructurada, a fin de recopilar información relevante acerca de lo que es importante y significativo en la mente de los participantes.

La entrevista constaba de una serie de preguntas abiertas y ordenadas que la investigadora iba realizando a los pacientes en ese orden, permitiendo en todo momento la libertad y flexibilidad en las respuestas (Anexo I). Se propició un clima lo más sereno, tranquilo e íntimo posible, para que el entrevistado se sintiese cómodo en todo momento. La duración media de cada entrevista era de 10-15 min, respecto a la recopilación de la información, cada entrevista fue grabada y posteriormente transcrita para su análisis e interpretación, realizando las anotaciones de campo necesarias en cada caso.

La recogida de datos fue llevada a cabo por la investigadora, en la misma sala donde en ocasiones previas se había realizado la consulta de enfermería, en concreto el despacho de Nefrología de las Consultas Externas del Hospital Universitario Reina Sofía, durante los meses de Abril y Mayo de 2014, atendiendo a la disponibilidad del entrevistado, sala, y en las franjas horarias en las que estaba activa la consulta.

\section{Consideraciones éticas}

A fin de respetar en todo momento los principios éticos de participación en este tipo de estudios, se contó con el Consentimiento Verbal de cada participante, exponiendo la finalidad y el propósito de estudio, garantizando en todo momento la privacidad y el anonimato de los entrevistados, así como la confidencialidad de las informaciones.

\section{RESULTADOS}

Sensibilización acerca del riesgo cardiovascular al que están expuestos y conocimiento de la propia enfermedad

A través del análisis de las entrevistas realizadas, se comprobó que verdaderamente la ES mejoró los conocimientos de la enfermedad en la población, en este caso hipertensa, puesto que al compararlos con los resultados obtenidos en los test iniciales, el resultado es mejor. No obstante, ayudándonos de las subcategorías que aparecieron dentro de esta categoría temática, se observó que la mejora existe, pero no es demasiado significativa.

\section{Motivo desencadenante de la enfermedad}

A la hora de atribuir a una causa, su problema de HTA, prácticamente la mitad de la muestra, tiene en cuenta factores desencadenantes modificables como el exceso de peso y el estrés. Resultó Ilamativo, que ninguno de ellos mencionase el factor herencia.

(Entrevistado 14) "En mi caso en concreto lo atribuiría a mi situación personal, sufro mucho estrés y me pasa factura.... aunque a día de hoy la tengo controlada, menos mal".

(Entrevistado 8) "Pues mira, yo creo que es por el estrés que tengo en mi día a día, pero según los entendidos es por el exceso de peso".

(Entrevistado 1) "Creo que lo de tener la tensión alta es por no regular la comida".

Frente a un número considerable que mantienen que su hipertensión está arraigada a cualquier otro problema físico o enfermedad, sin tener en cuenta los demás factores, o bien desconocen su causa.

(Entrevistado 3) "Yo no sé porqué la tengo alta, eso el médico lo sabrá ¿no?" (Entrevistado 16) "La hipertensión la tengo por mi problema de riñón" (Entrevistado12) "Mi hipertensión es consecuencia de mi problema oncológico, tengo cáncer".

\section{Percepción del riesgo al que se exponen}

La mayoría de los entrevistados conocían el riesgo al que están expuestos solo por ser un paciente con los niveles de presión arterial no controlados. De entre todos ellos, quizás por ser el más frecuente, todos coincidieron en el riesgo cardiovascular. Siendo citadas por tanto, complicaciones como: Infarto Agudo de Mliocardio, Ictus, Trombosis Venosa Profunda...

(Entrevistado 5) "Es grave, porque te puede dar un infarto, a mi me dio uno y estoy ahora con sintrom". 
(Entrevistado 10) "Tener la tensión alta es grave, vamos yo sé que te puede hacer daño en el corazón".

(Entrevistado 1) "Tener la tensión alta es algo grave porque podría darte alguna trombosis, un infarto cerebral...".

\section{Emociones y sentimientos}

Cuando el paciente aumenta su conocimiento, y comprueba que verdaderamente se expone a un riesgo bastante alto, de sufrir alguna complicación o desarrollar una enfermedad grave, aparecen en él una serie de emociones y sentimientos, entre las cuales destacaríamos "miedo", "temor" y "preocupación" (10 entrevistados). En contraposición a ello, al tratarse de una enfermedad "indolora" por así decirlo, y con una clínica prácticamente ausente, salvo en casos de HTA complicada, existe un número considerable de pacientes que muestran "despreocupación", "indiferencia" y "dejadez", ya que ven estas complicaciones como algo muy lejano e improbable (8 entrevistados).

(Entrevistado 1) "Lo que me asusta es precisamente eso, que me pase algo". (Entrevistado 5) "Al pronto me lo tomé bastante mal, me asusté... la tenía muy descontrolada y... me preocupaba que pudiese darme algo".

(Entrevistado 11) "La verdad que no me preocupa demasiado...".

(Entrevistado 15) "Al enterarme no reaccioné ni bien ni mal, no sé, tampoco es que me preocupe".

\section{Propuesta de tratamiento ideal para HTA}

Esta subcategoría surgió como respuesta a si tras la intervención sanitaria, el paciente hipertenso había interiorizado que el tratamiento no tiene porque ser solo farmacológico y que el no farmacológico, es igual o más importante. La mayoría mantuvo que el tratamiento ideal "son las pastillas" y pocos tienen en cuenta los hábitos higiénico-dietéticos.

(Entrevistado 16) "El que tengo de pastillas me va bien".

(Entrevistado 14) "El que tengo de pastillas yo diría que me va bien, pero como aportación personal propondría complementos como poner actividades relajantes, yoga, taichí....pero claro, eso no lo cubre el seguro".

(Entrevistado 12) "El adecuado sería aquel que combine pastillas y dieta, desde mi punto de vista. Yo ahora además, tengo que estar a dieta también por el azúcar, tú ya sabes... tengo muchos problemas, soy muy completita".

\section{Razonando los motivos que impulsan o retraen al paciente hipertenso a adherirse al tratamiento}

Una vez analizadas las entrevistas, se comprobó que existen una serie de factores que influyen en el régimen terapéutico. Con la salvedad que, dependiendo si el paciente se ha adherido o no al tratamiento no farmacológico, actúan como factores facilitadores (motivan al paciente a seguir), o por el contrario, se trata de factores que dificultan la adherencia.

Por tanto, fue necesaria la distinción entre adherentes y no adherentes para la descripción de esta categoría. Obteniendo por tanto que:

\section{a) Paciente hipertenso ADHERENTE:}

\section{- Factores facilitadores:}

- El apoyo familiar o apoyo "en casa" es un pilar fundamental para que el paciente se vea capaz de cambiar sus hábitos no saludables. Añaden que sobre todo en algunos aspectos en concreto como la dieta, cuando todos ponen de su parte, todo resulta mucho más fácil.

(Entrevistado 3) "No creo que se necesite apoyo, es un problema personal, que tienes que solucionar tú contigo mismo".

(Entrevistado 17) "Aunque creo que es más esfuerzo y voluntad propia, tengo el apoyo de mi mujer en casa".

- Resultó llamativo la unanimidad respecto a la importancia de la relación con el equipo sanitario, destacando una buena interrelación médicoenfermera-paciente, que incluya comunicación abierta, apoyo, ánimo, educación, seguridad...

(Entrevistado 12) "Por supuesto, la relación y el trato que tengan los profesionales contigo te influyen mucho a nivel emocional. Es necesario tener una buena relación".

(Entrevistado 11) "Para mí es muy importante, hay que ser profesional y saber estar en tu trabajo, hoy día no se calla nadie y lo veo bien verás... que sepamos lo que tenemos, no como antiguamente que los médicos...".

- En lo que respecta a las características de la personalidad, mantienen que es fundamental 
cambiar de actitud, que no es algo fácil pero que se puede conseguir.

(Entrevistado 15) "Hombre no es fácil pero se intenta".

(Entrevistado 12) "Bueno digamos que soy una persona muy positiva, y creo que es lo más importante, la actitud de uno mismo, más que el apoyo".

- Autoestima/Percepción propia, buena autoestima, se sienten bien. Ver como pierden peso y llegan a controlar los niveles de presión arteriales algo satisfactorio.

(Entrevistado 14) "Me siento muy bien, mejor ahora que hace 20 años, con eso te lo digo todo".

(Entrevistado 11) "La verdad que fenomenal, estoy a gusto conmigo mismo".

\section{- Factores que dificultan el manejo del régimen terapéutic 0 :}

- Los pacientes hipertensos afirman que seguir una dieta para mejorar la HTA supone un gasto de dinero.

(Entrevistado 11) "Hombre algo de dinero sí, para comer sano y variado se necesita dinero".

(Entrevistado 6) "Claro que sí, aunque bueno también hay cosas sanas y baratas, no todo es caro".

\section{a) Paciente hipertenso NO ADHERENTE:}

\section{- Factores facilitadores:}

- La importancia de la relación con el equipo sanitario es apreciada por todos los pacientes. Afirman que es algo primordial, de cara al tratamiento de una enfermedad.

(Entrevistado 18) "Siempre una buena relación ayuda a seguir y afrontar los problemas".

(Entrevistado 9) "Por supuesto, la forma con la que te explican las cosas, te dicen lo que te puede pasar...y claro, hacen que te lo pienses...pero...luego es muy difícil".

- De todos los no adherentes, ocho dicen saber que Io más importante en el tratamiento de la HTA, en concreto en sus circunstancias, es cambiar los hábitos higiénico-dietéticos.

(Entrevistado 4) "Creo que lo más importante es cambiar".
(Entrevistado 13) "Lo más importante y lo más difícil es cambiar los hábitos de vida".

- A excepción de uno, que da la misma importancia al tratamiento farmacológico, como no farmacológico, y otro que mantiene que el farmacológico es el que vale.

(Entrevistado 18) "Yo diría que no, que lo más importante es el tratamiento farmacológico".

(Entrevistado 10) "Creo que los dos son importantes verás...pero que ya te digo que...".

\section{Factores que dificultan el manejo del régimen terapéutico:}

- Les falta apoyo familiar, o no valoran el que tienen, tomándoselo a mal en la mayoría de los casos y llegando incluso a agobiarles.

(Entrevistado 10) "El apoyo es necesario, y a mí me falta mucho...tengo muchos problemas en casa y no puedo centrarme en mí".

(Entrevistado 1) "Mi mujer y mis hijos me animan a que cambie, pero la mayoría de las veces no lo valoro, me lo tomo a mal e incluso me enfado".

(Entrevistado 7) "No creo que me falte apoyo, porque más que tengo ya...lo que pasa que me lo salto a la torera".

- Falta de voluntad, se manifiesta en todos los pacientes, el cambio para ellos es algo imposible y muy difícil de lograr.

(Entrevistado 18) "La verdad que es muy difícil, pero yo creo que es más de cabeza".

(Entrevistado 8) "Si yo lo intento, pero es que no puedo, y sé que lo hago mal pero es que...".

- Se asoció el seguimiento de una dieta con un gasto de dinero importante, que en algunos casos es la justificación para no seguirla, aunque cuando la falta de voluntades el factor principal para no seguirla, el gasto de dinero lo ven irrelevante.

(Entrevistado 1) "No creo que sea necesario gastar demasiado dinero para seguir una dieta".

(Entrevistado 8) "No porque mira, hay productos sanos que son muy baratos como el pollo o el conejo".

- Autoestima/ Percepción propia: en su mayoría se ven bien y se sienten cómodos consigo mismos 
por lo que nada les motiva a cambiar. Excepcionalmente, cuatro pacientes indican que les gustaría cambiar pero no pueden, por problemas familiares, depresión, o simplemente físicos.

(Entrevistado 7) "Me siento bien, y si uno está contento consigo mismo le sobra todo lo demás".

(Entrevistado 9) "No me siento mal, pero me doy cuenta que no soy el mismo de antes porque hay cosas que no puedo hacer ya... y mira que no soy viejo, o al menos yo no me considero".

(Entrevistado 2) "Pues la verdad que me siento bastante mal, estoy pasando una racha con depresión y...".

\section{Relación entre cumplimiento del tratamiento farmacológico y no farmacológico}

Esta categoría surgió como respuesta a nuestro último objetivo específico: Determinar la relación entre el cumplimiento de las medidas farmacológicas y no farmacológicas. De antemano se piensa que si un paciente es cumplidor del tratamiento farmacológico, será cumplidor también del no farmacológico.

El número de pacientes de entre los no adherentes, que sin embargo es cumplidor del tratamiento farmacológico, es muy similar al de los adherentes a ambos tratamientos.

(Entrevistado 7) "No, nunca se me olvida, aunque sí que a la misma hora siempre no me la tomo".

(Entrevistado 10) "No no, yo siempre me la tomo".

En cuanto al número de pacientes de entre los adherentes a las medidas higiénico-dietéticas, que no cumplen el tratamiento farmacológico, es muy similar al de los no adherentes a ambos tratamientos.

(Entrevistado 3) "Bueno alguna vez lo mismo si que se me ha olvidado...".

(Entrevistado 4) "No siempre, pero puede que en alguna ocasión sí".

\section{Discusión}

Existen varios estudios que muestran cómo la efectividad de la ES en la mejora del cumplimiento terapéutico y los conocimientos sobre la HTA. Algunos autores, añaden que al utilizar técnicas de intervención mediante refuerzo conductual o con técnicas de apoyo familiar, aumentan el conocimiento y se mantiene a los 6 meses y 5 años, respectivamente ${ }^{6}$.
En contraposición a ello, otros autores obtienen resultados distintos en estudios donde se aplican técnicas similares, afirmando en este caso que no observan diferencias en el cumplimiento autocomunicado tras un ensayo con una técnica informativa y participativa (Mata,1987), o bien que solo mejora el cumplimiento durante el período de estudio (McKenney, 1973), incluso que aumentó el cumplimiento respecto al grupo control en los dos primeros años, perdiéndose el efecto cuando transcurrieron 5 años, tras intervenir con una dinámica de grupo (Morisky, 1983)6, 15,16,17.

En nuestro estudio, coincidimos en afirmar que tras la intervención realizada sobre los pacientes, existe una mejora en el conocimiento de la enfermedad, así como sus causas, riesgos...etc. Pero sin embargo, no implica una mejora significativa en la adherencia al tratamiento, dado que, del total de pacientes que componían la muestra, menos de la mitad de ellos, los que tras la intervención, deciden adherirse al tratamiento no farmacológico y cambiar al menos, dos de los factores desencadenantes modificables.

En lo que respecta al "cumplimiento o adherencia" al tratamiento farmacológico, objeto de estudio de gran número de publicaciones, nuestros resultados coinciden con lo publicado hasta el momento ${ }^{7,8,11}$. Las cifras de incumplimiento terapéutico en España son altas, aunque desde hace unos años se ha ido observando un progresivo descenso de las mismas 9 . Como se observa en nuestros resultados, del total de pacientes hipertensos entrevistados, una cuarta parte de los pacientes estudiados, afirman haber olvidado tomar su medicación en alguna ocasión, incluyendo pacientes adheridos y no adheridos al tratamiento no farmacológico.

Atendiendo a la sensibilización que muestran los pacientes acerca de los riesgos a los que se exponen si no "se cuidan" y controlan su enfermedad, tras haber recibido ES, podemos decir que efectivamente, se ha conseguido alertar a los pacientes de los posibles riesgos que implica tener HTA. La mayoría citan complicaciones como: "infarto agudo de miocardio, ictus, trombosis venosa profunda...", riesgo cardiovascular. Sin embargo, coincidiendo en este caso con los resultados expuestos en el estudio realizado en el Hospital Clinic de Barcelona, en el que la mayoría de los pacientes asociaban "riesgo" a problemas cardiacos y nadie, o muy pocos asocian la HTA a daño en otro tipo de órgano como puede ser el riñón o cerebro ${ }^{1}$.

Entre los hallazgos de nuestro estudio, resulta llamativo que el total de los pacientes considerasen muy importante la relación que se establece con 
el equipo sanitario de cara a abordar una enfermedad. Afirman que tener una buena relación y un trato cordial con el mismo, es un punto a favor para seguir el régimen terapéutico, favorece que se sientan cómodos para expresar sus dudas y miedos, saben que en todo momento van a estar al tanto de su evolución y eso les da confianza y se sienten apoyados; lo que a fin de cuentas contribuye a que se vean motivados a "hacer las cosas bien".

Por otro lado, es conocido que en la adherencia terapéutica existen varios factores psicosociales que influyen, tales como: las creencias, las actitudes, las atribuciones, la representación mental de la enfermedad y el apoyo social ${ }^{18,19}$. Si lo comparamos con los datos obtenidos en nuestro estudio, se podría afirmar que, coincidiendo con las mismas, existen una serie de factores que influyen en el paciente a la hora de adherirse al tratamiento, motivándolo o retrayéndolo en cada caso. Consideramos factores facilitadores, aquellos que hacen más sencilla la adherencia, y factores que dificultan el manejo del régimen terapéutico, a los que suponen un impedimento para la misma.

Es curioso que un mismo factor, pueda valorarse de forma tan diferente dependiendo de si la persona decide adherirse 0 no al tratamiento. Un ejemplo evidente de ello lo tenemos con el "apoyo familiar y social", siendo valorado como algo positivo y de agradecer, entre los pacientes que intentan cambiar, o por el contrario, como algo negativo, agobiante e incluso causa de enfado en algunas ocasiones, para aquellos que no deciden adherirse.

Por ello, podríamos decir que lo fundamental al realizar una intervención educativa, es individualizar y personalizar la intervención, explorando los conocimientos de los que se parte y los puntos débiles del paciente, para hacer un mayor hincapié en ellos, trabajando juntos, resolviendo dudas y dando el apoyo necesario para solventar las deficiencias. La ES tiene que ser siempre bidireccional, si no se convierte en mera información. Con el objetivo de que el paciente se sienta capacitado para cambiar sus malos hábitos, con el esfuerzo y la voluntad que requiere. Es cierto que para llevar a cabo dicha tarea, se requiere muchísimo más tiempo y dedicación que para dar algunas nociones básicas sobre buenos hábitos higiénico-dietéticos, pero posiblemente esa no sea la cuestión, sino falta de dedicación, déficit de habilidades y preparación en el terreno pedagógico, $y$ tal vez psicosocial.

\section{Implicaciones para la práctica}

Como implicaciones para la práctica, los resultados muestran la necesidad de que el profesional de enfermería adquiera competencias específicas como educador sanitario.

Es fundamental además, personalizar la intervención educativa, pues las motivaciones, actitudes y capacidades de cada paciente son tan variables que una misma educación no sirve para todos.

Es indispensable la intervención psicológica en aqueIlos pacientes, en los que el problema supera a los conocimientos adquiridos, pues hay que modificar su actitud ante "la vida".

\section{Limitaciones de estudio}

Los propios de los estudios cualitativos y la escasa bibliografía publicada sobre el tema. Existen pocos estudios publicados en España que demuestren la eficacia de programas educativos para pacientes con HTA, que puedan servir de guía para los profesionales sanitarios.

A la vista de estos resultados podemos concluir que:

- La ES impartida, es beneficiosa para el paciente hipertenso puesto que mejora su nivel de conocimientos sobre la enfermedad y lo sensibiliza acerca del riesgo que ésta conlleva.

- La falta de adherencia al tratamiento no farmacológico, se manifiesta por una falta de voluntad entre los pacientes hipertensos refractarios, más que por un problema de información, siendo necesaria una intervención por parte del personal de enfermería enfocado a solventar la misma.

- Ser un buen cumplidor del tratamiento farmacológico no implica buena adherencia a las medidas higiénico-dietéticas y viceversa.

- Finalmente, decir que la relación que el equipo sanitario mantiene con el paciente, hipertenso en este caso, es básica para el afrontamiento necesario de los inconvenientes asociados a la enfermedad.

Recibido: 20 octubre 2014

Revisado: 25 octubre 2014

Modificado: 25 octubre 2014

Aceptado: 25 octubre 2014 


\section{Bibliografía}

1. Estrada, D., Jiménez, L., Pujol, E.,\& De la Sierra, A. (2002). Nivel de conocimientos de los pacientes hipertensos ingresados en un servicio de medicina sobre su hipertensión y el riesgo cardiovascular. Consultada el 13-04-14. Clínica Barcelona España [disponible en la web] <http://www. fac.org.ar/tcvc/llave/ t|010/t|010.PDF>.

2. Nuñiz J, Juane R. La hipertensión arterial en España. Rev. Esp. Cardiol, 1995; 48 (Supl4):3-6.

3. Fernández, L., Guerrero, L., Segura, J., \& Gorostidi, M. Papel del personal de enfermería en el control de la hipertensión arterial y en la investigación cardiovascular. Hipertensión y Riesgo Vascular; 2010, 27, 41-52.

4. Rebollo P, Fernández-Vega F, Ortega. La evaluación de la calidad de vida relacionada con la salud en enfermos con hipertensión arterial. Hipertensión; 2001, 18:429-439.

5. Daugherty, S. L., Powers, J. D., Magid, D. J., Tavel, H. M., Masoudi, F.A., Margolis, K. L \& Ho, P.M. Incidence and prognosis of resistant hypertension in hypertensive patients. Circulation; 2012, 125(13), 1635-1642.

6. Márquez Contreras, E., Casado Martínez, J.J., Celotti Gómez, B., Gascón Vivó, J., Martín de Pablos, J.L., Gil Rodríguez, R \& Domínguez, R. El cumplimiento terapéutico en la hipertensión arterial. Ensayo sobre la intervención durante 2 años mediante educación sanitaria. Atención primaria; 2000, 26(1), 5-10.

7. Puigventos Latorre $F$, Llodrà Ortolà $V$, Vilanova Boltó M, Delgado Sánchez 0, Lázaro Ferreruela M, Forteza-Rey Borrallerás Jetal. Cumplimiento terapéutico en el tratamiento de la hipertensión: 10 años de publicaciones en España. Med. Clin (Barc) 1997; 109: 702-706.

8. Márquez Contreras, E., Martín de Pablos, J.L., Gil Guillén, V., Martel Claros, N., Motero Carrasco, J., \& Casado Martínez, J.J. La inercia clínica profesional y el incumplimiento farmacológico: ¿cómo influyen en el control de la hipertensión arterial? Estudio CUMAMPA. Hipertensión; 2008, 25(5),187-193.

9. Márquez Contreras, E., Gil Guillén, V., Casado Martínez, J.J., Martel Claros, N., de la Figuera Von Wichmann, M., Martín de Pablos, J. L, \& Espinosa García, J. Análisis de los estudios publicados sobre el incumplimiento terapéutico en el tratamiento de la hipertensión arterial en España entre los años 1984 y 2005. Atención primaria; 2006, 38(6), 325-332.

10. Márquez Contreras, E., De la Figuera Von Wichmann, M., Gil Guillén, V., Ylla-Catalá, A., Figueras, M., Balaña, M., \& Naval, J. Eficacia de una intervención informativa a hipertensos mediante mensajes de alerta en el teléfono móvil (HTA- ALERT). Atención primaria; 2004, 34(8),399-405.

11. Navarro J, Bonet A, Gosalbes V. Perspectiva del cumplimiento terapéutico en el tratamiento de la enfermedad crónica cardiovascular en España a partir del análisis bibliométrico y temático de su producción científica (1969-1999). Med. Clin (Barc) 2001; 116(supl.2):147-51.

12. Hayness R B, McKibbon K A, Kanani R, Bronwets $M$ C, Oliver T. Interventions for helping patients to follow prescriptions dor medications (Cochrane Review). The Cochrane Library Issue 1. Oxford: Update Sofware, 1999.

13. Hayness R B, Wang E, Da Mota Gomes M. A critical review of interventions for improve adherence with prescriber medications. Patients Educ Consel 1987; 10:155-66.

14. Mayan, M., \& Introductoria, N. Una introducción a los métodos cualitativos. Módulo de entrenamiento para estudiantes y profesionales. Alberta: International Institute for Qualitative Methodology (2001); 34.

15. Mata Cases, M., Monraba Capella M, Travería Solas M, Guarner M A, Antó Boque J M, Guarner M A. Papel de la educación sanitaria en la mejora del cumplimiento de los pacientes hipertensos en asistencia primaria. Aten. Primaria 1987; 4:189-194.

16. McKenney J M, Slining J M, Henderson H R, Devins $D$, Barr M. The effect of clinical pharmacy services on patients with essential hypertension. Circulation 1973; 48:1104-1111.

17. Morisky D E, Levine D M, Green DW. Five year blood pressure control and mortality following health education for hypertensive patients. Am J Public Health $1983 ; 2: 153-162$.

18. Cisneros, N. S. Adherencia terapéutica en hipertensión arterial sistémica. Revista mexicana de enfermería cardiológica; 2006, 14(3), 98-101.

19. Orueta S R. Estrategias para mejorar la adherencia terapéutica en enfermedades crónicas. Información terapéutica del Sistema Nacional de Salud 2005; 2(29); 40-45. 
Anexo 1. Análisis de los factores que determinan la adherencia terapéutica del paciente hipertenso. Entrevista semi-estructurada. Metodología cualitativa.

\section{a. Conocimiento sobre la enfermedad.}

1. ¿Cuál piensa usted que es la causa de su presión alta?

2. ¿Desde cuándo la padece?

3. ¿Cómo se tomó eso de tener la presión alta, alguna reacción?

4. ¿Qué clase de tratamiento cree que deba recibir?

5. ¿Piensa que tener la presión alta es algo grave? ¿Me podría explicar por qué si o no?

6. Me interesaría saber qué le asusta o preocupa respecto a la presión arterial elevada.

\section{b. Adherencia al tratamiento farmacológico.}

7. Algunas personas olvidan tomar sus medicamentos diariamente, ¿le sucede a usted también?

8. ¿Tiene alguna regla para recordar que tiene que tomar sus pastillas?

\section{c. Cumplimiento del tratamiento no farmacológico.}

9. ¿Vive solo en casa? ¿Quién prepara la comida?

10. ¿Come con sal?

11. ¿Considera que seguir una dieta adecuada para controlar la HTA requiere de un gasto monetario importante?

12. ¿Cuida su dieta para no engordar? ¿Sabría decirme las razones que le impiden perder peso?

13. ¿Es fumador?........ $n^{\circ}$ de cigarrillos diarios, ¿Bebe alcohol?..........Frecuencia......

14. Sinceramente, ¿Qué piensa que es más importante: seguir el tratamiento farmacológico 0 cambiar los malos hábitos de vida?

15. ¿Le han explicado en la consulta que si perdiera peso e hiciese ejercicio, igual no necesitaría la pastilla?

16. ¿Hace ejercicio regularmente? ¿Cómo se siente después?

17. ¿Cree que es importante la actitud y el carácter del personal sanitario a la hora de tratar la enfermedad? ¿Cómo es su relación con su equipo sanitario (médico/enfermera)?

18. ¿Siente que le falta apoyo para seguir hábitos saludables?

19. ¿Cómo se siente usted consigo mismo?

20. ¿Le gustaría hablarme de algo que no hayamos tratado? 